\title{
Segunda opinião em Oftalmologia
}

\author{
Second opinion in Ophthalmology
}

\author{
Wilson Nahmatallah Obeid ${ }^{1}$ \\ Luiz Antônio Vieira ${ }^{2}$ \\ Assad Youssef Frangieh ${ }^{3}$
}

\begin{tabular}{|l|}
\hline RESUMO \\
\hline Objetivo: Avaliar a porcentagem de pacientes oftalmológicos que buscam \\
uma segunda opinião e verificar o principal motivo dessa busca. Métodos: \\
A metodologia da pesquisa consiste na entrevista direta de 806 pacientes \\
oftalmológicos, atendidos em hospital especializado, por meio de um \\
questionário elaborado, com variáveis que podem estar relacionadas à \\
busca da segunda opinião. Resultados: Observou-se que 32\% dos pacien- \\
tes oftalmológicos solicitam a segunda opinião e destes, 31\% procuram \\
outros médicos para confirmar diagnóstico. Conclusão: A busca pela \\
segunda opinião em Oftalmologia está diretamente vinculada, em sua \\
maioria, à questão da relação médico/paciente. Tal situação serve de alerta \\
para o atual ensino profissional, apontando a necessidade de dedicação, \\
empenho e maior investimento no aprendizado, tanto dos atuais como dos \\
futuros profissionais, no que diz respeito a essa questão. Ressalta-se \\
também, a necessidade de melhorar as condições atuais de remuneração \\
e trabalho. Talvez, a revisão desses conceitos possa contribuir para a \\
diminuição desses problemas. \\
\hline
\end{tabular}

Descritores: Relações médico-paciente; Referência e consulta; Pacientes/psicologia; Aceitação do paciente de cuidados de saúde; Questionários

\section{INTRODUÇÃO}

O termo segunda opinião refere-se ao direito do paciente de determinar o seu tratamento, isto é, o direito de receber, selecionar o tratamento e o médico de sua preferência ${ }^{(1-2)}$. É um termo usado pelos serviços médicos para designar uma consulta adicional com outro médico ou grupo de médicos, solicitada pelo paciente ou pelo profissional que o está atendendo ${ }^{(3)}$.

Devido o crescente nível de conscientização e conhecimentos dos pacientes quanto aos seus problemas de saúde, complexidade científica e técnica da medicina, problemas legais e econômicos associados à prática médica, as atividades de segunda opinião estão ficando cada vez mais importantes ${ }^{(3)}$.

Em um artigo que descreve sobre a segunda opinião médica, relata que as comunidades notaram que algo interessante se incorporava à medicina. O programa de segunda opinião era inovador e aplicável às cirurgias, despertando o interesse e estimulando a cooperação dos médicos. Vários cirurgiões e grupos de segunda opinião foram instituídos em inúmeras cidades e estados dos Estados Unidos da América (EUA) ${ }^{(4)}$.

A maioria das pesquisas sobre segunda opinião foi realizada em pacientes que necessitavam de procedimentos cirúrgicos. Entretanto, poucas estudaram fatores psicológicos determinantes para a sua procura ${ }^{(5)}$.

No Japão, o aumento dessa conscientização trouxe mudança de hábitos. Com uma atitude passiva na interação com os médicos, os pacientes estão mais informados quanto aos seus direitos de saber do seu estado de saúde, 
medicação e tratamento utilizados, reservando-se o direito de procurar ajuda adicional (segunda opinião) $)^{(2)}$.

Alguns autores referem que a procura de segunda opinião está associada a sintomas crônicos, inabilidade por parte dos médicos em explicar, ou por parte dos pacientes em entender o problema, incerteza de diagnóstico e tratamento por parte do profissional, e até mesmo um certo grau de alterações psiquiátricas diagnosticadas nos pacientes. O motivo mais comum é a persistência dos sintomas e a dificuldade em manter uma relação longa (consulta) entre médico e paciente ${ }^{(5)}$.

Durante a revisão da literatura, apenas a gastroenterologia e a ortopedia foram especialidades que pesquisaram os motivos psicológicos que levaram os pacientes a procurar uma segunda opinião ${ }^{(1,5-6)}$. Dentre os artigos pesquisados, não houve relatos em oftalmologia que pudessem ser comparados com esses autores. Com isso, há um interesse em investigar, na área oftalmológica, o número de pacientes que procura a segunda opinião e os motivos psicológicos que os levam a isso. Pode-se assim verificar se a área em questão também proporciona aos pacientes, fatores que os levem a esse tipo de comportamento.

\section{OBJETIVOS}

1. Pesquisar através de questionário, a freqüência de pacientes que ficam insatisfeitos com o primeiro médico oftalmologista consultado e procura uma segunda opinião.

2. Pesquisar através de questionário, o motivo que gera a insatisfação com a primeira consulta e leva esses pacientes a procurarem a segunda opinião.

\section{MÉTODOS}

A pesquisa foi realizada na cidade de São Paulo (SP), no Hospital CEMA, situado na zona leste, que é referência em oftalmologia e otorrinolaringologia para esta região. Porém, recebe pacientes de todas as regiões da cidade e do Estado de São Paulo.

Este hospital está dividido em duas partes físicas: uma destinada aos pacientes do Sistema Único de Saúde (SUS) e outra para os particulares e conveniados. Nessa última, pacientes pertencentes ao SUS são atendidos esporadicamente. Possui equipes especializadas em refração, glaucoma, doenças externa e córnea, retina, plástica ocular, estrabismo, uveíte, trauma ocular, ecografia, visão subnormal, neuroftalmologia e pronto atendimento.

Serão entrevistados 806 pacientes sendo divididos em 2 grupos: grupo 1 (pacientes que procuram uma segunda opinião) e grupo 2 (pacientes que não procuram uma segunda opinião).

A abordagem do paciente será realizada no corredor de acesso à saída do hospital, onde o entrevistador não tem conhecimento de qual consultório ou recepção que o paciente haverá saído. $\mathrm{O}$ entrevistador se dirigirá ao paciente solicitando sua atenção e explicando o questionário. Após o paciente conceder a permissão, se iniciará as perguntas, sendo realiza- das de maneira clara e objetiva. O questionário vai ser mostrado ao entrevistado de forma que este também possa ler e responder às questões. A cada pergunta realizada e respondida, o entrevistador, sem solicitar uma explicação ou induzir a qualquer outra resposta, apenas marcará a resposta dada pelo entrevistado, iniciando-se, a seguir, uma outra pergunta.

Todos os pacientes pertencem ao ambulatório de oftalmologia e as perguntas serão sempre realizadas em relação à mesma doença (citada pelo paciente como a causa da consulta).

O pesquisador realizará perguntas relacionadas ao sexo (masculino ou feminino); idade ( 0 a 15 anos, 16 a 30 anos, 31 a 45 anos, 46 a 60 anos e > 60 anos); categoria de atendimento (Sistema Único de Saúde, particular ou convênio); forma de atendimento (hora marcada ou pronto-atendimento); motivo da consulta (glaucoma, refração, doenças externa e córnea, retina, estrabismo, pronto-atendimento e plástica ocular); local que originou a segunda consulta (próprio hospital ou externo); tempo de duração dos sintomas em relação à mesma doença ( $<30$ dias ou > 30 dias); forma de encaminhamento (iniciativa própria, indicação de familiares ou amigos, indicação de convênio, indicação de médico externo, já conhecia, propaganda e outros); motivo que o levou a procurar outro médico (não confiou, médico desinteressado, confirmar diagnóstico e conduta, ou não soube responder).

Quanto à faixa etária, todos os pacientes incluídos e aqueles que tinham dificuldade de comunicação eram auxiliados pelos acompanhantes.

Em relação à forma de atendimento, os pacientes poderiam ser atendidos de três formas: particular, convênio ou através do SUS. Os pacientes pertencentes ao SUS eram atendidos esporadicamente.

A forma de atendimento é realizada de duas maneiras: hora marcada ou pronto-atendimento. $\mathrm{O}$ paciente da hora marcada pode agendar sua consulta por telefone ou pessoalmente, escolhendo o médico ou subespecialidade que preferir. O paciente do pronto-atendimento pode se consultar a qualquer hora.

Quando perguntado o motivo da consulta, os pacientes relatavam suas queixas que poderiam estar relacionadas com os seguintes tipos de doença. As expressões usadas pelos pacientes foram:

- glaucoma: "glaucoma" e "pressão alta nos olhos";

- refração: "refração", "astigmatismo", "miopia" e "hipermetropia";

- doenças externa e córnea: "córnea", "blefarite" e "problema de córnea";

- retina: "retina", "retinopatia diabética", "descolamento de retina";

- estrabismo: "estrabismo" e "olho torto";

- pronto-atendimento: "batida nos olhos", "dor no olho", "conjuntivite" e "irritação";

- plástica ocular: "plástica ocular", "tumor de pálpebra", "verruga" e "canal da lágrima".

O local de origem da segunda opinião poderia ser o próprio hospital, ou consultórios, clínicas e hospitais externos.

Quanto ao tempo de doença, foram considerados tempo inferior a 30 dias ou maior que 30 dias. 
Em relação à forma de encaminhamento, o paciente pode chegar ao hospital das seguintes maneiras: iniciativa própria, indicação de familiares ou amigos, indicação de convênios, já conhecia, propaganda ou outros.

Quanto à razão que levou os pacientes a procurar outro médico, esta era relatada espontaneamente pelos pacientes. Com isso, as queixas foram divididas em quatro categorias, colocando as expressões relatadas pelos pacientes quando entrevistados:

- não confiou no médico: "não confiou", "não confiou em que o médico disse", "não sentiu confiança nos atos profissionais", "não confiou na explicação médica", "não sentiu segurança" e "falta de confiança nos médicos",

- médico desinteressado: "achou desatencioso", "achou grosso", "pouca atenção no seu caso", "faltou ânimo no profissional" e "profissional sem paciência",

- confirmar diagnóstico e conduta: "confirmar a doença que tem", "confirmar conduta e diagnóstico" e "confirmar tratamento e diagnóstico",

- não soube responder .

A pesquisa foi realizada na parte que atende pacientes particulares e conveniados.

Todos os pacientes encaminhados por médicos externos (não pertencentes ao corpo clínico do hospital) ou passaram pela primeira vez em consulta ou procuraram atendimento por outro motivo (doença) não foram considerados pertencentes ao grupo de segunda opinião.

A carta de informação sobre a pesquisa e o termo de consentimento foram aplicados para cada paciente entrevistado.

A análise estatística utilizada foi baseada no teste quiquadrado com nível de significância de 5\%.

\section{RESULTADOS}

A seguir, apresentaremos os resultados obtidos nesta pesquisa, que serão ilustrados por meio de tabelas.

Foram questionados 806 pacientes pertencentes à área oftalmológica em um hospital privado, na zona leste de São Paulo. Destes, 256 (32\%) solicitaram uma segunda opinião.

O principal motivo que leva o paciente a procurar uma segunda opinião é para confirmar diagnóstico e conduta.

A doença do paciente, em relação à desconfiança ou falta de interesse do primeiro médico, está relacionada com o motivo da busca por uma segunda opinião $(\mathrm{p}=0,002)$. $\mathrm{O}$ motivo de procurar uma segunda opinião é estatisticamente a mesma para

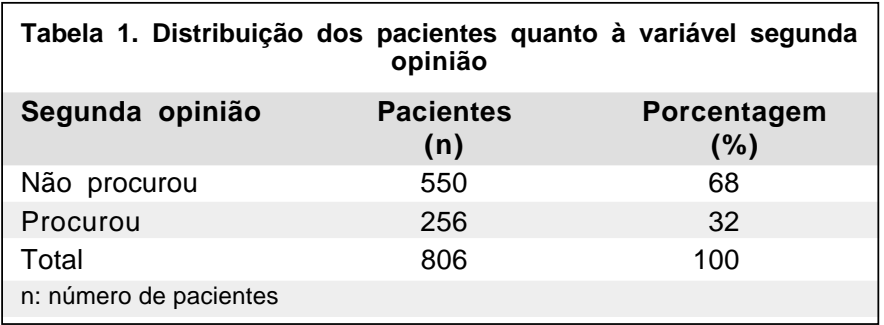

quem tem doença relacionada a glaucoma, refração, doença externa e córnea, retina ou estrabismo $(\mathrm{p}=0,328)$ e também é a mesma para quem tem queixa relacionada à catarata, pronto atendimento ou plástica ocular $(\mathrm{p}=0,456)$. Porém, os pacientes que apresentam doenças relacionadas a glaucoma, refração,

\begin{tabular}{|lcc|}
\hline \multicolumn{3}{|c|}{$\begin{array}{l}\text { Tabela 2. Distribuição dos pacientes que procuram a segunda } \\
\text { opinião e souberam responder sobre o motivo da procura }\end{array}$} \\
$\begin{array}{l}\text { Motivo de procurar } \\
\text { outra opinião }\end{array}$ & $\begin{array}{c}\text { Pacientes } \\
\text { (n) }\end{array}$ & $\begin{array}{c}\text { Porcentagem } \\
(\%)\end{array}$ \\
$\begin{array}{l}\text { Desconfiança no } \\
\text { primeiro médico }\end{array}$ & 60 & 33 \\
$\begin{array}{l}\text { Achou os profissionais } \\
\text { sem interesse }\end{array}$ & 42 & 24 \\
$\begin{array}{l}\text { Confirmar diagnóstico } \\
\text { e conduta }\end{array}$ & 78 & 43 \\
$\begin{array}{l}\text { Total } \\
\text { n: número de pacientes }\end{array}$ & 180 & 100 \\
\hline
\end{tabular}

\begin{tabular}{|c|c|c|c|c|c|c|}
\hline \multirow[t]{2}{*}{ Doença } & \multicolumn{2}{|c|}{$\begin{array}{l}\text { Desconfiança no } \\
\text { primeiro médico ou } \\
\text { achou os profissio- } \\
\text { nais sem interesse }\end{array}$} & \multicolumn{2}{|c|}{ Outros } & \multicolumn{2}{|c|}{ Total } \\
\hline & $\mathbf{n}$ & $\%$ & $\mathbf{n}$ & $\%$ & $\mathbf{n}$ & $\%$ \\
\hline Glaucoma & 24 & 45 & 30 & 55 & 54 & 100 \\
\hline Refração & 30 & 45 & 36 & 55 & 66 & 100 \\
\hline D. externa e córnea & 14 & 54 & 12 & 46 & 26 & 100 \\
\hline Retina & 12 & 40 & 18 & 60 & 30 & 100 \\
\hline Estrabismo & 10 & 71 & 4 & 29 & 14 & 100 \\
\hline Catarata & 8 & 24 & 26 & 76 & 34 & 100 \\
\hline Pronto-atendimento & 2 & 10 & 18 & 90 & 20 & 100 \\
\hline Plástica ocular & 2 & 17 & 10 & 83 & 12 & 100 \\
\hline Total & 102 & 40 & 154 & 60 & 256 & 100 \\
\hline
\end{tabular}

\begin{tabular}{|c|c|c|c|c|c|c|}
\hline \multirow[t]{2}{*}{ Doença } & \multicolumn{2}{|c|}{$\begin{array}{c}\text { Confirmar } \\
\text { diagnóstico } \\
\text { e conduta } \\
\end{array}$} & \multicolumn{2}{|c|}{ Outros } & \multicolumn{2}{|c|}{ Total } \\
\hline & $\mathbf{n}$ & $\%$ & $n$ & $\%$ & $\mathbf{n}$ & $\%$ \\
\hline Glaucoma & 18 & 33 & 36 & 67 & 54 & 100 \\
\hline Refração & 22 & 33 & 44 & 67 & 66 & 100 \\
\hline D. externa e córnea & 6 & 23 & 20 & 77 & 26 & 100 \\
\hline Retina & 12 & 40 & 18 & 60 & 30 & 100 \\
\hline Estrabismo & 0 & 0 & 14 & 100 & 14 & 100 \\
\hline Catarata & 12 & 35 & 22 & 65 & 34 & 100 \\
\hline Pronto-atendimento & 6 & 30 & 14 & 70 & 20 & 100 \\
\hline Plástica ocular & 2 & 17 & 10 & 83 & 12 & 100 \\
\hline Total & 78 & 30 & 178 & 70 & 256 & 100 \\
\hline
\end{tabular}


doenças externa e córnea, retina ou estrabismo procura mais por uma segunda opinião devido à desconfiança no médico ou porque acharam os profissionais sem interesse que pacientes que apresentam alteração relacionada à catarata, pronto-atendimento ou plástica ocular $(\mathrm{p}<0,001)$.

Não foi observada nenhuma significância entre as patologias quanto à confirmação de diagnóstico e conduta.

A doença do paciente, em relação a variável confirmar diagnóstico e conduta, não está relacionada com motivo da busca por uma segunda opinião $(\mathrm{p}=0,188)$.

\section{DISCUSSÃO}

A maioria das pesquisas sobre segunda opinião foi realizada em pacientes encaminhados para cirurgias. Entretanto, poucas estudaram os fatores psicossociais determinantes que contribuem para isso. Geralmente, o paciente aceita o diagnóstico realizado na primeira consulta e sente-se satisfeito, porém, às vezes, solicita uma confirmação com outro especialista ${ }^{(5)}$.

Já se têm notícias de que, no Japão, o crescimento de associações de pacientes propiciou uma mudança passiva na interação médico/paciente. Os pacientes estão mais conscientes sobre seus direitos de obter informações pertinentes à sua saúde, medicação e tratamentos empregados, bem como de participar da decisão médica. Essa consciência faz com que eles optem por uma ajuda adicional, isto é, uma segunda opinião $0^{(5-7)}$.

$\mathrm{O}$ presente estudo analisou, através de um questionário elaborado (Anexo 1), fatores que podem contribuir para a busca de uma segunda opinião, em se tratando de pacientes oftalmológicos.

Foram entrevistados 806 pacientes e observamos que 256 (32\%) solicitaram uma segunda opinião.

Os pacientes que optaram por uma segunda opinião procuraram dois ou mais médicos, semelhante ao encontrado por outro artigo que apontou $30 \%{ }^{(8)}$.

Autores relataram no Japão, dos 1.033 pacientes pesqui- sados, $420(40,6 \%)$ procuraram um segundo médico e 226 $(21,9 \%)$ mais de dois médicos. Nessa análise, foram enviados questionários via correio para os pacientes, que preenchiam e reenviavam ${ }^{(5)}$.

Outra pesquisa mostrou que $20(7,5 \%)$ dos 266 pacientes avaliados pela gastroenterologia procuraram mais de um médico. No Canadá $16 \%$ dos pacientes solicitaram uma segunda opinião e incluíram a medicina alternativa (acupuntura) como forma de tratamento ${ }^{(1)}$. Tal variação pode estar relacionada com as diferentes especialidades pesquisadas ou com as variações e peculiaridades culturais de cada país.

Neste trabalho, os motivos que levaram os pacientes a uma segunda opinião foram, respectivamente, confirmar diagnóstico e conduta (31\%), não soube responder (30\%), desconfiança no primeiro médico (23\%), achou os profissionais desinteressados $(16 \%)$.

Em outra análise, excluímos a variável em que os pacientes não souberam responder, apresentando novos dados: confirmar condutas e diagnósticos (43\%), desconfiaram do primeiro médico (33\%), acharam o profissional sem interesse (24\%). Autores demonstram que $30 \%$ dos pacientes procuram um segundo médico para confirmar diagnóstico e conduta e $60 \%$ ficam insatisfeitos com a primeira consulta. Nesse trabalho, ao se constatar a insatisfação do paciente, o autor refere que 50\% relatam que o primeiro médico não demorou tempo suficiente para consulta ${ }^{(4)}$. Em outro trabalho, o autor relata que $43 \%$ dos pacientes que procuraram a segunda opinião foram para confirmar diagnóstico e conduta e 33\% ficaram insatisfeitos com o primeiro médico ${ }^{(5)}$. Portanto, tais dados se aproximam aos observados neste trabalho.

Em nosso trabalho, pacientes com estrabismo, doenças externa e córnea, glaucoma, refração ou retina procuram mais a segunda opinião. Os pacientes referem ter desconfiança nos profissionais ou os acham desinteressados pela sua doença.

Quando solicitaram uma confirmação de diagnóstico e conduta, não foram observados dados significativos entre as

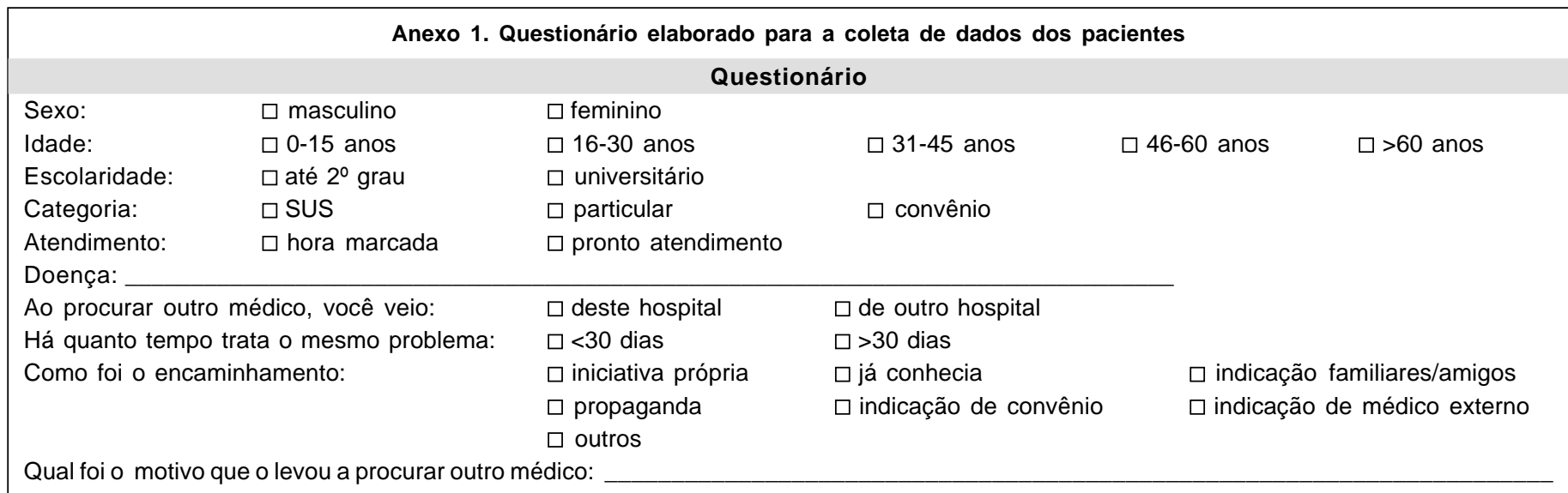


doenças. Essa variável, mesmo não sendo significativa entre as doenças, apresentou um alto índice de confirmação de diagnóstico e conduta. Fatores que podem influenciar tal panorama podem estar relacionados com a formação acadêmica do profissional, sobre o conhecimento de doenças, o que dificulta a orientação dos pacientes.

A variável sexo não está relacionada com a busca da segunda opinião. Esse resultado é semelhante ao encontrado por outros autores.

Pacientes de até 15 anos procuram menos outro médico (22\%) do que aqueles de 16 anos ou mais (27\% a 39\%), diferindo dos resultados encontrados por outros pesquisadores que não referiram diferenças entre as idades ${ }^{(1)}$.

A variável escolaridade não está relacionada com a procura de outro médico. Este resultado é semelhante ao descrito por alguns investigadores ${ }^{(1)}$. Outro artigo referiu que, no Japão, os pacientes que procuraram um segundo médico e aqueles que ficaram satisfeitos com o primeiro têm o nível de escolaridade maior do que aqueles que optaram pela busca de mais de duas opiniões ${ }^{(5)}$.

Pacientes com hora marcada geram em $40 \%$ dos casos a consulta adicional.

Em relação à categoria de atendimento, notou-se, estatisticamente, que a busca da segunda opinião ocorre com a mesma frequiência para as categorias particular (31\%) ou convênio (33\%).

Pacientes que apresentam doenças com tempo superior a 30 dias solicitam mais a segunda opinião (42\%). Estes resultados são semelhantes aos encontrados em outras pesquisas ${ }^{(1-2,5)}$.

Os familiares ou amigos influenciaram $48 \%$ dos pacientes do grupo de segunda opinião na procura de ajuda adicional, diferindo de outros achados onde os pacientes não sofreram influência dos familiares ou amigos ${ }^{(1)}$.

Demonstramos, em relação às doenças, a porcentagem de pacientes entrevistados, em análise descritiva, evidenciando a refração como o ambulatório com maior número de atendimento (24\%), seguida pelo pronto-atendimento (21\%).

Pacientes que apresentam alterações relacionadas à plástica ocular procuram mais a segunda opinião (67\%). A menor incidência é no ambulatório de pronto-atendimento (12\%).

Os hospitais, clínicas ou consultórios externos (estabelecimentos não pertencentes ao hospital pesquisado) foram responsáveis por $73 \%$ dos casos de segunda opinião, enquanto que, o hospital estudado originou apenas $27 \%$ dos casos de segunda opinião.

Quando relacionamos o local de origem da segunda opinião, com o tipo de ambulatório, observamos que o pronto-atendimento foi o setor que originou menos a consulta adicional, porém, quando esta ocorre, tem o hospital pesquisado $(60 \%$ dos casos) como o local de origem. $\mathrm{O}$ fato de originar maior número de segunda opinião pode ser explicado por fatores como o maior tempo de espera a que esses pacientes estão sujeitos, atendimento rápido, proporcionando um menor contato com o médico, dificultando a realização de perguntas e obten- ção de respostas. Isso está de acordo com outra pesquisa que demonstrou um grande índice de insatisfação ocorre quando os pacientes relatam que o tempo gasto na consulta não foi suficiente para perguntar e obter as respostas desejadas ${ }^{(1)}$. Em relação às outras doenças, observamos que a segunda opinião originou-se em hospitais, clínicos ou consultórios externos.

A satisfação durante uma consulta é relatada através do relacionamento entre as expectativas do paciente e suas experiências ${ }^{(6)}$.

As experiências com serviço de saúde podem ter impacto direto nas expectativas do paciente sobre o serviço ${ }^{(7)}$. Embora nem todos os pacientes possam ter esse impacto, referem o que pensam, o que vão receber, o que desejam e intitulam o que acham ser importante na procura do atendimento ${ }^{(8)}$.

$\mathrm{O}$ relacionamento entre expectativas e experiências nem sempre é direto, mas quando a experiência diverge substancialmente das expectativas, a insatisfação é o principal resultado ${ }^{(9)}$.

Um levantamento realizado pelo Conselho Regional de Medicina do Estado de São Paulo, de 12.000 denúncias registradas entre 1995 a 2001, relacionou as principais queixas dentro das dez especialidades que mais receberam denúncias. São respectivamente, ginecologia, pediatria, ortopedia e traumatologia, medicina do trabalho, oftalmologia, cirurgia plástica, cardiologia, psiquiatria, gastroenterologia e infectologia. De acordo com esse levantamento, as principais queixas foram negligência, imperícia e imprudência; atendimento médico; conduta ético-profissional; relação médico/paciente; perícia médica; publicidade médica; condições de funcionamento do hospital; relações entre médicos; atestado médico; honorários médicos. Diante desses dados, observa-se que a relação médico/paciente é a quarta categoria com maior número de queixas ${ }^{(10)}$.

\section{CONCLUSÕES}

O número de pacientes que fica insatisfeito com o primeiro médico oftalmologista consultado e procura uma segunda opinião é de $32 \%$.

A desconfiança em $33 \%$ dos casos é o principal motivo que gera a insatisfação dos pacientes com a primeira consulta e os levam a procurar a segunda opinião.

\section{ABSTRACT}

Purpose: To evaluate the percentage of ophthalmologic patients who search for a second opinion and to verify the main reason for this search. Methods: The methodology of the present study consists of a direct interview with 806 ophthalmologic patients, attended at a specialized hospital, through an adequately elaborated questionnaire, containing variables that can be related to the search for this second opinion. Results: It was observed that $32 \%$ of the ophthalmologic patients ask for a second opinion and, of these, $31 \%$ search for other physicians in order to confirm the diagnosis. Conclusion: Most search for the second opinion in Ophthalmology is directly linked to the 
doctor/patient relationship. Such situation serves to warn the present professional teaching, pointing to the need for dedication, effort and greater investment in learning, regarding both the present and the future professionals. In addition, it is important to emphasize the necessity in improving the working conditions and wages. Maybe, the review of these aspects can contribute to decrease the mentioned problems.

Keywords: Referral and consultation; Physician-patient relations; Patients/psychology; Patient acceptance of health care; Questionnaires

\section{REFERÊNCIAS}

1. Sutherland LR, Verhoef MJ. Patients who seek a second opinion: are they different from the typical referral? J Clin Gastroenterol. 1989;11(3):308-13.
2. Sutherland LR, Verhoef MJ. Why do patients seek a second opinion or alternative medicine? J Clin Gastroenterol. 1994;19(3):194-7.

3. Sabbatini RME. O direito à segunda opinião médica. J1 Correio Popular (Campinas). [periódico on-line] 2000 [citado 2000 Abril 14]; (Abril 14): [2 telas]. Disponível em: http://www.cpopular.com.br

4. McCarthy EG, Widmer GW. Effects of screening by consultants on recommende elective surgical procedures. N Engl J Med. 1974;291:1331-5.

5. Sato T, Takeichi M, Hara T, Koizumi S. Second opinion behavior among Japanese primary care patients. Br J Gen Pract. 1999;49(444):546-50.

6. Kronz JD, Westra WH, Epstein JI. Mandatory second opinion surgical pathology at a large referral hospital. Cancer. 1999;86(11):2426- 35. Comment in: Cancer. 1999;86(11):2198-200.

7. Donabedian A. The definition of quality and approaches to its assessment. Michigan: Health Administration Pre; 1980. (Exploration in quality assessment and monitoring, 1).

8. Nogueira RP. Conceitos e princípios para programas de gestão de qualidade em serviços de saúde: Rio de Janeiro: Qualitymark, 1993.

9. Vuori H. El control de calidad en los serviços: conceptos y metodologia. Barcelona: Masson; 1988.

10. Conselho Regional de Medicina do Estado de São Paulo. Guia da relação médico/paciente. São Paulo: CREMESP; 2001. 48p. 